\title{
Ratcheting response of spherical vessels under load-controlled condition based on the Chaboche viscoplastic model
}

\author{
Vahid Falahi ${ }^{\text {iD }}$, Hossein Mahbadi*2 iD, Mohammad Reza Eslami ${ }^{1}$ iD \\ ${ }^{1}$ Amirkabir University of Technology, Department of Mechanical Engineering, Tehran, Iran \\ ${ }^{2}$ Islamic Azad University, Department of Mechanical Engineering, Tehran, Iran
}

\begin{abstract}
In this study, ratcheting behavior of thick spherical vessels subjected to mechanical cyclic loads at elevated temperature, using the Chaboche unified viscoplastic model with combined kinematic and isotropic theory of plasticity, is investigated. Since this model is rate dependent, loading rate plays a crucial role on the ratcheting responses. A precise and general numerical procedure, using the modified successive approximation iterative method of solution to solve the non-linear equations, is developed to obtain the cyclic inelastic creep and plastic strains. Effects of loading and unloading rate, inside pressure, thickness of vessel, creep time and environmental temperature on ratcheting responses, and stress amplitude of the vessel due to the inside pressure cyclic loading at elevated temperature are obtained. The ratcheting response is observed for the load-controlled conditions, as investigated in this paper. It is shown that increasing the loading and unloading rates and the thickness of pressure vessels, result into decrease in the ratcheting rate while increasing the inside pressure, creep time, and temperature distribution increase the ratcheting rate. Also, stress amplitude decreases with increasing the creep time and thickness of vessel. On the other hand, increasing the loading and unloading rate, inside pressure, and temperature distribution result into increasing the stress amplitude. The results obtained using the applied method in this study is verified with the experimental data given in the literature. By simplifying the constitutive model, numerical results are compared with the finite elements results.
\end{abstract}

\section{Keywords}

Cyclic loading; Thick pressure vessels; Creep; Viscoplastic hardening model; Time dependent plasticity; Ratcheting strain

Received: 28 February 2020; Accepted: 04 May 2020

ISSN: 2630-5763 (online) C 2020 Golden Light Publishing All rights reserved.

\section{Introduction}

Since many structures that are widely used in industry and power plants are subjected to cyclic loading, the study of these structures is crucial and important in the structural design problems. In some applied cases, the load might be increased enough to bring the structure into plastic range. As a result, the accumulated strain plays an acute role in design problems. In special cases, the structures are used in high temperature environments by which the structure shows viscoplastic behavior.

* Corresponding author

Email: h_mahbadi@iauctb.ac.ir 
Thus, the investigation of structural failure in order to predict lifetime of structure and allowable loading rate is significantly important.

Numerous constitutive models to estimate the ratcheting behavior of structures are proposed in literature. In order to evaluate the plastic responses of structures, the isotropic, kinematic, and combined hardening models are proposed. Hill [1] and Westergaard [2], respectively, published abstract of researchers' works related to the isotropic hardening theory. Base on this method, the radius of yield surface is extended in the HaighWestergaard stress plane. Inelastic analysis of circular rod based on the isotropic hardening model using the von Mises yield criterion is discussed by Neal [3].

Due to the Baushinger effect, however, there is a deficiency in isotropic hardening model. According to the Baushinger effect, yield surface is transferred in the Haigh-Westergaard stress space, where this translation is not considered in the isotropic hardening model. The first kinematic hardening model capable to consider translation of the yield surface was proposed by Prager [4]. This model basically falls into the linear kinematic hardening theory. In order to precisely evaluate plastic responses, Armstrong and Frederick [5] proposed a nonlinear kinematic hardening model. The nonlinear term in this model makes the slope of strain-stress curve different in loading and unloading process and as a result cyclic ratcheting behavior is predict more accurately and correctly. A kinematic hardening model for cyclic plasticity was proposed by Chaboche [6], which uses a combination of three terms of the ArmstrongFrederick model in order to coincide the experimental data and the predicted results more accurately. Plastic cyclic behavior of thick pressure vessels using the kinematic hardening criterion is studied by Mahbadi and Eslami [7]; the paper compares the Prager linear kinematic hardening with the Armstrong-Frederick nonlinear kinematic hardening model. It is concluded that the nonlinear theory of the later model can correctly predict ratcheting responses of structure. Also, Komijani et al. [8] and Ejtemajou et al. [9] have investigated cyclic behavior of thick spherical and cylindrical, respectively, vessels made of transversely isotropic materials using the anisotropic yield criterion and have concluded that the Armstrong-Frederick hardening model predicts ratcheting behavior under load controlled cyclic loading and reversed plasticity under strain controlled condition while Prager hardening model anticipates reversed plasticity for both strain and stress controlled condition.

A time independent constitutive theory for cyclic plasticity using combined isotropic and kinematic hardening model is proposed by Chaboche [10] to improve the evaluation of cyclic behavior of structures. According to this model, the radius of yield surface is either increased or transferred in the stress space spontaneously. In contrast to this rate independent hardening model, Chaboche $[11,12]$ proposed a time dependent unified viscoplastic model using viscoplastic potential which is capable to evaluate effects of loading rate and creep time. As like as Chaboche [10], this constitutive model is a combination of isotropic and kinematic hardening models. Also, another viscoplastic hardening models are proposed in order to estimate the inelastic responses of structures exposed to elevated temperatures such as Walker [13], Murakami and et al. [14], Moreno and Eric [15], Ohno [16] and Lee and Krempl [17]. The most significance of these viscoplastic constitutive theories is how to determine material constants which are used in these models. Multiple experimental tests, such as Mahnken and Stein [18], Schwertel and Schinke [19], Fossum [20, 21], Tong et al. [22], Zhan [23] and Gong et al. [24] are done in order to calculate these parameters. Plastic responses of beams under cyclic loading based on the time independent Chaboche hardening model has been investigated by Shojaei [25]. A modified constitutive model has been proposed based on the unified viscoplasticity theory considering timedependent kinematic hardening for relaxation by Wufan Chen et al. [26]. Dao-Hang Li et al. [27] have investigated a modified unified viscoplastic constitutive model in order evaluate axial torsional thermos mechanical cyclic loading. A series of 
experiments has been conducted by Wufan Chen et al. [28] to determine creep and fatigue behavior of 316 stainless steel and to revisit a unified viscoplastic model. Benaarbia et al. [29] developed a thermodynamically-based viscoelasticviscoplastic model for the high temperature cyclic behavior of $9-12 \% \mathrm{Cr}$ steels considering isotropic and kinematic hardening. Rae et al. [30] studied experimental characterization and computational modelling of cyclic viscoplastic behavior of turbine steel and accumulated strain fields has been investigated under realistic thermos-mechanical fatigue loading. The rate-dependent ratcheting characteristics of 35CRMo alloy have been investigated under cyclic uniaxial tension considering different stress amplitudes at high temperature by Zheng [31]. There are also other papers deal with viscoplastic constitutive models such as those studied by Dong [32], Feng [33], Szmytka [34], Kyaw [35], Ahmed [36], Luk-Cyr [37] and XiaoAn [38].

In this paper, the Chaboche unified viscoplastic model is used to evaluate the cyclic behavior of thick spherical pressure vessels due to the mechanical cyclic loading. This model is a rate dependent constitutive theory with combined isotropic and kinematic hardening models. The considered spherical vessel is made of 316 stainless steel under isothermal condition at temperature 500 ${ }^{\circ} \mathrm{C}$. The material parameters are obtained from Gong [24]. The precise numerical method based on a modified successive approximation proposed by Mahbadi et al. [7-9, 25] is developed and used to estimate the viscoplastic cyclic loading of thick vessels. The feature of the present work that makes it unique in comparison with the published papers on cyclic loading of thick vessels is the time dependency of this viscoplastic model which makes it capable to indicate effects of loading rate and relaxation time.

\section{Mathematical Formulation}

Consider a thick spherical vessel made of isotropic materials with inside and outside radii $R_{1}$ and $R_{2}$ subjected to inside and outside pressures $P_{1}$ and $P_{2}$, respectively. In order to achieve convergence, the parameters are normalized. The dimensionless parameters are considered in Eq. (1).

$$
\begin{aligned}
& \bar{\sigma}_{r r}=\frac{\sigma_{r r}}{\sigma_{0}}, \bar{\sigma}_{\theta \theta}=\frac{\sigma_{\theta \theta}}{\sigma_{0}}, \bar{\sigma}_{\phi \phi}=\frac{\sigma_{\phi \phi}}{\sigma_{0}}, \bar{\epsilon}_{r r}=\frac{\epsilon_{r r}}{\epsilon_{0}}, \\
& \bar{\epsilon}_{\theta \theta}=\frac{\epsilon_{\theta \theta}}{\epsilon_{0}}, \bar{\epsilon}_{\phi \phi}=\frac{\epsilon_{\phi \phi}}{\epsilon_{0}}, \bar{\epsilon}_{r r}^{v p}=\frac{\epsilon_{r r}^{v p}}{\epsilon_{0}}, \bar{\epsilon}_{\theta \theta}^{v p}=\frac{\epsilon_{\theta \theta}^{v p}}{\epsilon_{0}}, \\
& \bar{\epsilon}_{\phi \phi}^{v p}=\frac{\epsilon_{\phi \phi}^{v p}}{\epsilon_{0}}, \bar{\epsilon}_{r r}^{\text {Res }}=\frac{\epsilon_{r r}^{\text {Res }}}{\epsilon_{0}}, \bar{\epsilon}_{\theta \theta}^{\text {Res }}=\frac{\epsilon_{\theta \theta}^{R e s}}{\epsilon_{0}}, \bar{\epsilon}_{\phi \phi}^{\text {Res }}=\frac{\epsilon_{\phi \phi}^{\text {Res }}}{\epsilon_{0}}, \\
& \bar{P}_{1}=\frac{P_{1}}{\sigma_{0}}, \bar{P}_{2}=\frac{P_{2}}{\sigma_{0}}, \rho=\frac{r}{R_{1}}, \beta=\frac{R_{2}}{R_{1}}
\end{aligned}
$$

In Eq. (1), $\sigma_{i j}$ is the stress tensor and $\epsilon_{i j}$ is the strain tensor. The subscripts $r r, \theta \theta$ and $\phi \phi$ represent radial, tangential, and meridional directions, respectively. Here, $v p$ and Res superscripts indicate viscoplastic strain and residual strain, respectively. $\sigma_{0}$ is the initial yield stress and $\epsilon_{0}$ is the initial yield strain. The dimensionless equilibrium, compatibility, and stress-strain relations of considered spherical vessel are, respectively, as

$$
\begin{aligned}
& \bar{\sigma}_{r r, \rho}-\frac{2\left(\bar{\sigma}_{\theta \theta}-\bar{\sigma}_{r r}\right)}{\rho}=0 \\
& \bar{\epsilon}_{\theta \theta, \rho}-\frac{\bar{\epsilon}_{r r}-\bar{\epsilon}_{\theta \theta}}{\rho}=0 \\
& \bar{\epsilon}_{r r}=\bar{\sigma}_{r r}-v\left(\bar{\sigma}_{\theta \theta}+\bar{\sigma}_{\phi \phi}\right)+\bar{\epsilon}_{r r}^{v p}+\bar{\epsilon}_{r r}^{R e s} \\
& \bar{\epsilon}_{\theta \theta}=\bar{\sigma}_{\theta \theta}-v\left(\bar{\sigma}_{r r}+\bar{\sigma}_{\phi \phi}\right)+\bar{\epsilon}_{\theta \theta}^{v p}+\bar{\epsilon}_{\theta \theta}^{\text {Res }}
\end{aligned}
$$

In Eq. (4), $v$ is Poisson's ratio. Due to the spherical symmetry, $\bar{\sigma}_{\theta \theta}=\bar{\sigma}_{\phi \phi}$ and $\bar{\epsilon}_{\theta \theta}=\bar{\epsilon}_{\phi \phi}$.

Substituting Eq. (4) into Eq. (3) and using equilibrium equation Eq. (2), a system of differential equations is found as

$$
\begin{aligned}
& (1-v) \bar{\sigma}_{\theta \theta, \rho}-v \bar{\sigma}_{r r, \rho}+\bar{\epsilon}_{\theta \theta, \rho}^{v p}+\bar{\epsilon}_{\theta \theta, \rho}^{\text {Res }} \\
& -(1+v) \frac{\bar{\sigma}_{r r}-\bar{\sigma}_{\theta \theta}}{\rho}-\frac{\bar{\epsilon}_{r r}^{v p}+\bar{\epsilon}_{r r}^{\text {Res }}}{\rho}+\frac{\bar{\epsilon}_{\theta \theta}^{v p}+\bar{\epsilon}_{\theta \theta}^{\text {Res }}}{\rho}=0 \\
& \bar{\sigma}_{r r, \rho}-\frac{2\left(\bar{\sigma}_{\theta \theta}-\bar{\sigma}_{r r}\right)}{\rho}=0
\end{aligned}
$$


By solving the above system of differential equations, radial and tangential stresses can be obtained as follow

$$
\begin{aligned}
\bar{\sigma}_{r r}= & \frac{1}{1-v} \int_{1}^{\rho} \frac{\bar{\epsilon}_{r r}^{v p}}{\rho} d \rho+\frac{1}{1-v} \int_{1}^{\rho} \frac{\bar{\epsilon}_{r}^{\text {Res }}}{\rho} d \rho+\frac{C_{2}}{\rho^{3}}+C_{1} \\
\bar{\sigma}_{\theta \theta}= & \frac{1}{1-v} \int_{1}^{\rho} \frac{\bar{\epsilon}_{r r}^{v p}}{\rho} d \rho+\frac{\bar{\epsilon}_{r r}^{v p}}{2(1-v)}+\frac{1}{1-v} \int_{1}^{\rho} \frac{\bar{\epsilon}_{r r}^{\text {Res }}}{\rho} d \rho \\
& +\frac{e_{r}^{\text {Res }}}{2(1-v)}+\frac{C_{2}}{\rho^{3}}-\frac{C_{2}}{2 \rho^{3}}+C_{1}
\end{aligned}
$$

In Eq. (5), $C_{1}$ and $C_{2}$ are constants of integration and are defined using boundary conditions. Boundary conditions are shown in Eq. (6).

$$
\begin{gathered}
\bar{\sigma}_{r r}(1)=-\bar{P}_{1} \\
\bar{\sigma}_{r r}(\beta)=-\bar{P}_{2}
\end{gathered}
$$

Using the boundary conditions of Eq. (6) and substituting into Eq. (5), constants $C_{1}$ and $C_{2}$ are determined as

$C_{1}=-\bar{P}_{1}-C_{2}$

$C_{2}=\frac{\beta^{3}}{\beta^{3}-1}\left(\bar{P}_{1}-\bar{P}_{2}-\frac{1}{1-v} \int_{1}^{\beta} \frac{\bar{\epsilon}_{r r}^{v p}}{\rho} d \rho-\frac{1}{1-v} \int_{1}^{\beta} \frac{\bar{\epsilon}_{r r}^{\text {Res }}}{\rho} d \rho\right)$

\section{Hardening Model}

In this paper, the unified viscoplastic hardening model proposed by Chaboche $[11,12]$ is considered to evaluate ratcheting behavior of thick pressure vessels at elevated temperatures. This model is a time dependent constitutive model which includes kinematic and isotropic kinematic hardening theories. The flow rule of this model using the von Mises yield criterion is shown in Eq. (8)

$\dot{\epsilon}_{i j}^{v p}=\frac{3}{2} \dot{\epsilon}^{v p} \frac{S_{i j}-A_{i j}^{\prime}}{J\left(\sigma_{i j}-A_{i j}\right)}$

Where $S_{i j}$ and $A_{i j}^{\prime}$ are the deviatoric stress and back stress tensors, $J$ is a distance in the stress space, which is given in Eq. (9).
$J\left(\sigma_{i j}-A_{i j}\right)=\sqrt{\frac{3}{2}\left(S_{i j}-A_{i j}^{\prime}\right)\left(S_{i j}-A_{i j}^{\prime}\right)}$

The following relation is used as yield criterion in this hardening model

$f=J\left(\sigma_{i j}-A_{i j}\right)-R-\sigma_{0}$

Rate of effective viscoplastic strain is defined as

$\dot{\epsilon}^{v p}=\left\langle\frac{J\left(\sigma_{i j}-A_{i j}\right)-R-\sigma_{0}}{Z}\right\rangle^{n}$

The McCauley bracket $\langle$.$\rangle is used here to ensure$ that when $f<0$, the state of stress is inside the elastic domain. In Eqs. (10) and (11), $n$ and $Z$ are material constants, $R$ is associated parameter to the isotropic hardening of material and is recalled as drag stress.

In this viscoplastic model, the back stress tensor, which related to kinematic hardening theory, is described in Eq. (12).

$\dot{A}_{i j}=\dot{A}_{i j}^{(1)}+\dot{A}_{i j}^{(2)}$

Where $\dot{A}_{i j}^{(1)}$ and $\dot{A}_{i j}^{(2)}$ are the non-linear kinematic hardening parameters which are obtained as

$\dot{A}_{i j}^{(1)}=C_{1}\left(a_{1} \dot{\epsilon}_{i j}^{v p}-A_{i j}^{(1)} \dot{\epsilon}^{v p}\right)$
$\dot{A}_{i j}^{(2)}=C_{2}\left(a_{2} \dot{\epsilon}_{i j}^{v p}-A_{i j}^{(2)} \dot{\epsilon}^{v p}\right)$

The parameters $C_{1}, C_{2}, a_{1}$ and $a_{2}$ are material constants for the Chaboche kinematic hardening model and are obtained from the uniaxial test. Drag stress associated with increasing the yield surface is defined as

$\dot{R}=b(Q-R) \dot{\epsilon}^{v p}$

The normalized quantities of hardening model are defined as

$$
\begin{aligned}
& \bar{f}=\frac{f}{\sigma_{0}}, \bar{C}_{k}=\frac{C_{k}}{E}, \bar{a}=\frac{a}{\sigma_{0}}, \bar{A}=\frac{A}{\sigma_{0}}, \\
& \bar{R}=\frac{R}{\sigma_{0}}, \bar{Z}=\frac{Z \epsilon_{0}^{1 / n}}{\sigma_{0}}, \bar{Q}=\frac{Q}{\sigma_{0}}, \bar{b}=\frac{b \sigma_{0}}{E}
\end{aligned}
$$




\section{Numerical Solution}

In this section, the numerical solution procedure for viscoplastic analysis and ratcheting behavior of thick spherical vessels under inside pressure cyclic loading is described. Since the relations are nonlinear and the inelastic strains depend upon the loading path, a numerical iterative procedure is proposed in the present work to evaluate the cyclic behavior of thick sphere. The numerical method which is proposed by Mahbadi et al. [7-9, 25] is developed for viscoplastic constitutive relations. This method is applied to the extension of Picard's method of the successive approximation to nonlinear equations.

The procedure for viscoplastic solution is as follow:

1. All the input quantities are normalized with the initial yield stress and elasticity modulus.

2. Total time is divided into $n$ steps and total load associated with the time division $(\operatorname{Load}=f(t))$ is divided into $m$ steps. Also, the cross section of thick sphere is divided into $p$ parts.

3. By increasing the time one step, the yield criterion is checked for all cross section parts and if that part is in elastic region the inelastic strain equals to zero. Otherwise, the inelastic strains must be obtained as follow.

4. A value is considered as the first approximation of equivalent inelastic strain increment.

5. Then, the components of inelastic strain increment is obtained using the flow rule by Eqs. (16) and added to the accumulation inelastic strain to obtain the total inelastic strain

$$
\begin{aligned}
& \Delta \bar{\epsilon}_{i j}^{v p}=\frac{3}{2} \bar{\epsilon}^{v p} \frac{\bar{\sigma}_{i j}-\bar{A}_{i j}}{\bar{J}} \\
& \bar{\epsilon}_{i j}^{v p}=\bar{\epsilon}_{i j}^{v p(a c c)}+\Delta \bar{\epsilon}_{i j}^{v p}
\end{aligned}
$$

It must be noted that since $\bar{J}, \bar{A}$, and $\bar{\sigma}$ parameters are dependent on inelastic strains, the values of previous time step are used in this step.

6. The back stress tensor related to kinematic hardening theory is obtained from Eq. (17).

$$
\begin{aligned}
& \Delta \bar{A}_{i j}^{(n)}=\bar{C}_{n}\left(\bar{a}_{n} \Delta \bar{\epsilon}_{i j}^{v p}-\bar{A}_{i j}^{(n)} \Delta \bar{\epsilon}^{v p}\right) \\
& \Delta \bar{A}_{i j}=\sum_{n=1}^{2} \Delta \bar{A}_{i j}^{(n)} \\
& \bar{A}_{i j}=\bar{A}_{i j}^{a c c}+\Delta \bar{A}_{i j}
\end{aligned}
$$

7. Isotropic hardening parameter, using the approximated inelastic strains in step 5, is obtained as

$$
\begin{aligned}
& \Delta \bar{R}=\bar{b}(\bar{Q}-\bar{R}) \Delta \bar{\epsilon}^{v p} \\
& \bar{R}=\bar{R}^{a c c}+\Delta \bar{R}
\end{aligned}
$$

8. Calculate the new values of stresses from Eqs. (5). A trapezoidal numerical integration is used to obtain the existence integrations.

9. Using the calculated quantities in step 8 , new inelastic strain increment can be obtained from Eq. (19).

$$
\Delta \bar{\epsilon}^{v p}=\left\langle\frac{\bar{J}\left(\bar{\sigma}_{i j}-\bar{A}_{i j}\right)-\bar{R}-1}{\bar{Z}}\right\rangle^{n} \Delta t
$$

10. The new value of inelastic strain increment must be compared with the initial guess and if the difference between these two values is small enough, repeat the method from steps 3 to 10 , otherwise steps 5 to 10 must be repeated until the convergence occurs. The following relative error is considered to determine convergency:

$$
\left|\frac{\Delta \bar{\epsilon}_{v p}^{\text {New }}-\Delta \bar{\epsilon}_{v p}^{\text {Previous }}}{\Delta \bar{\epsilon}_{v p}^{\text {New }}}\right|<10^{-7}(20)
$$

For unloading response, two different coordinate axes, Fig. 1, are defined at the end of loading and unloading curves and instead of obtaining the residual strains, loading process is applied. At the end of unloading process, the stresses and strains are transferred to the origin coordinate system. This method is used to assign the correct sign to the equivalent stress and strain. The following steps must be followed for unloading procedure. 


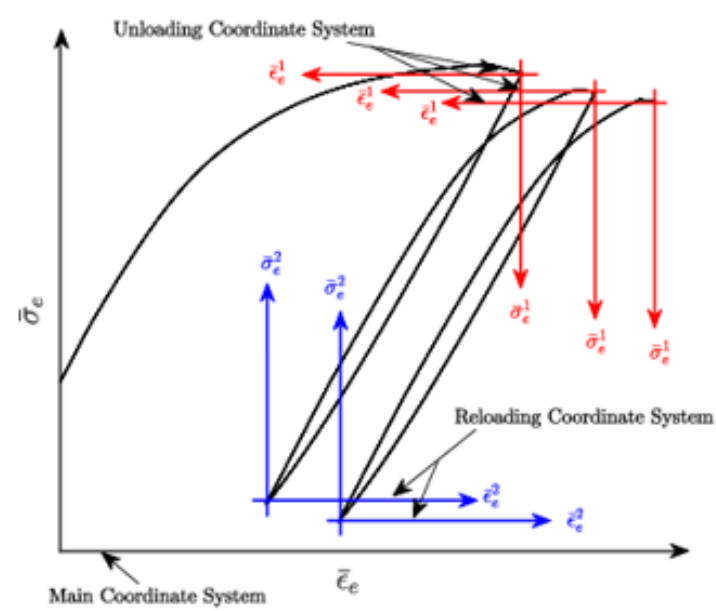

Fig. 1. Unloading and reloading coordinate systems

11. Set the strains components and the stress components to zero and transfer kinematic hardening parameters to unloading coordinate using the following transmission law

$\bar{A}_{i j}^{a c c}=\bar{\sigma}_{i j}^{(2)}-\bar{A}_{i j}$

Where $\bar{A}_{i j}$ is the accumulated back stress tensor in the unloading coordinate system and $\bar{\sigma}_{i j}^{(2)}$ is the maximum stress tensor in the loading coordinate system.

12. Steps 2 through 10 is repeated in order to evaluate the unloading responses. All the calculated quantities in the unloading coordinate systems are transferred to the main coordinate using the following transmission relation

$$
S_{i j}=\left(\sum_{k=1}^{m} S_{i j}^{L(k)}-\sum_{k=1}^{m-1} S_{i j}^{U L(k)}\right)-S_{i j}^{U L(m)}
$$

where $S_{i j}$ is any tensor including stress, total strain, inelastic strain, and back stress. Superscripts $L(k)$ indicate the final value of $S_{i j}$ in $k^{\text {th }}$ cycle of loading. Also, $U L(k)$ indicates the final value of $S_{i j}$ in $i^{\text {th }}$ cycle of unloading and $m$ shows the last cycle.
13. A new coordinate system in the same direction of main coordinate is considered in order to obtain the reloading responses. As same as unloading process, the strains components and the stress components are set to zero and kinematic hardening parameters are transferred to the reloading coordinate using the following transmission law

$$
\bar{A}_{i j}^{a c c}=\bar{\sigma}_{i j}^{(1)}-\bar{A}_{i j}
$$

14. Similar to unloading, steps 2 through 10 are repeated in order to estimate the reloading responses. All the calculated quantities in the reloading coordinate systems are transferred to the main coordinate using the following transmission relation

$$
S_{i j}=\left(\sum_{k=1}^{m-1} S_{i j}^{L(k)}-\sum_{k=1}^{m-1} S_{i j}^{U L(k)}\right)+S_{i j}^{U L(m)}
$$

15. The procedures between steps 2 through 15 are repeated until the final cycle of load is achived.

The flow chart corresponding to the proposed numerical procedure is illustrated in Fig. 2.

\section{Results and Discussion}

In this section the effect of different parameters such as loading and unloading rate, creep time, inside pressure, thickness of vessel, and temperature on the ratcheting behavior and stress amplitude at critical point of vessel is discussed. Some responses from other papers and finite element software are used in order to verify the numerical procedure. For the following examples, a thick spherical vessel made of SS316 steel is considered. The radius ratio of the vessel is $\beta=1.5$. The material properties based on the Chaboche viscoplastic constitutive model are given in Table 1 [24].

\subsection{Verification}

In Fig. 3, the numerical results obtained in present work are compared with experimental data for a beam made of 316 stainless steel [24] at uniform temperature $500{ }^{\circ} \mathrm{C}$. 


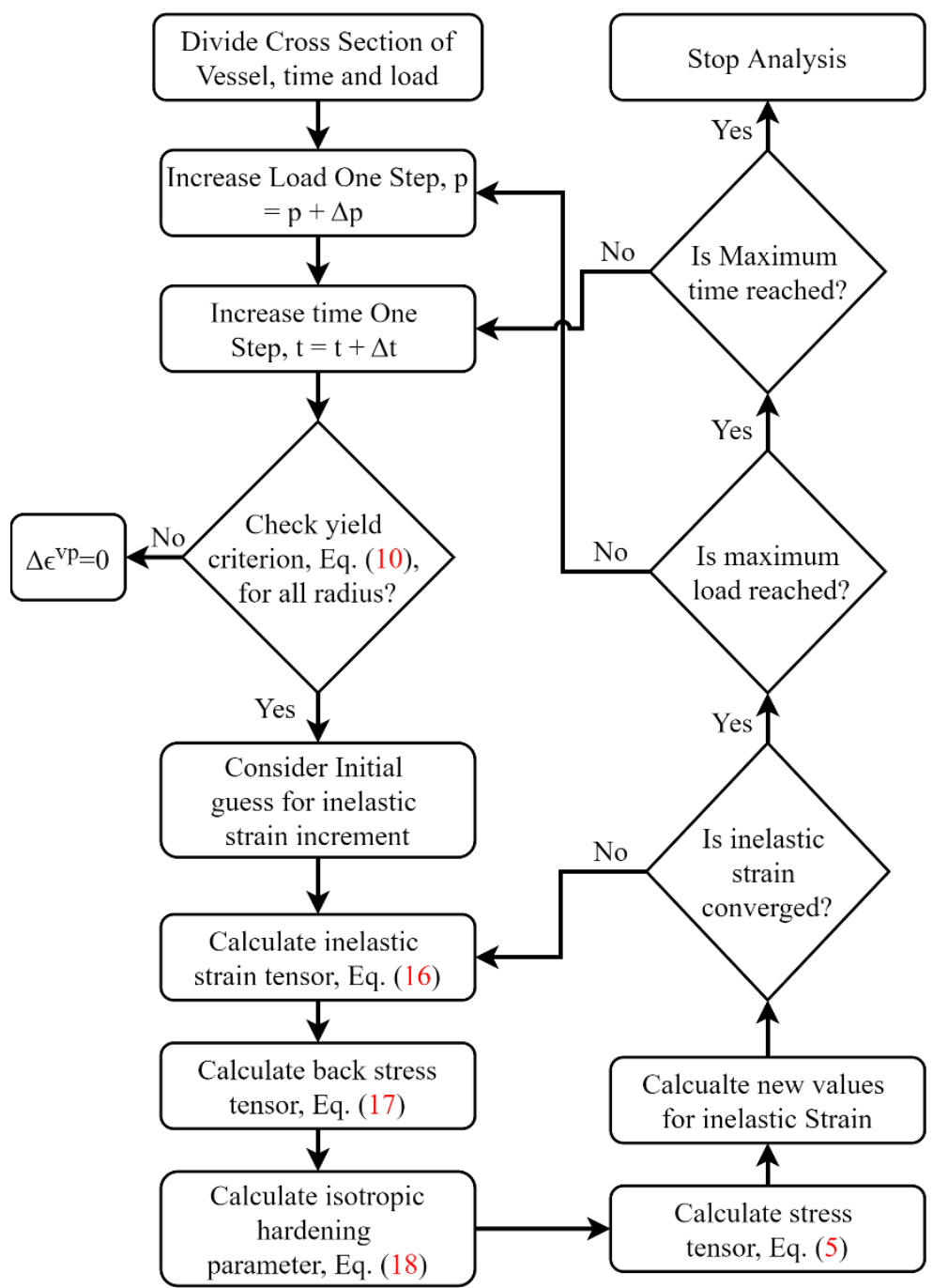

Fig. 2. Flow chart of proposed numerical procedure

Table 1. Material constant at multiple temperatures [24]

\begin{tabular}{lllll}
\hline \multirow{2}{*}{ Material property } & \multicolumn{3}{c}{ Temperature $\left({ }^{\circ} \mathrm{C}\right)$} \\
\cline { 2 - 5 } & 300 & 500 & 550 & 600 \\
\hline$\sigma(\mathrm{MPa})$ & 39 & 32.5 & 31 & 30 \\
$E(\mathrm{MPa})$ & 154.84 & 145.54 & 141.26 & 139.12 \\
$b$ & 39.46 & 33.35 & 31 & 28.6 \\
$Q(\mathrm{MPa})$ & 32.76 & 30.41 & 27.8 & 27.43 \\
$a_{1}(\mathrm{MPa})$ & 119.1 & 94.6 & 86.3 & 80.06 \\
$C_{1}$ & 5964.1 & 6472.6 & 6939 & 7111.9 \\
$a_{1}(\mathrm{MPa})$ & 108.4 & 113.3 & 114.8 & 116 \\
$C_{1}$ & 1001.6 & 979.91 & 957.69 & 928.7 \\
$Z \mathrm{MPa} \cdot \mathrm{s}^{1 / n}$ & 179 & 175 & 173 & 170 \\
$n$ & 10 & 10 & 10 & 10 \\
\hline
\end{tabular}




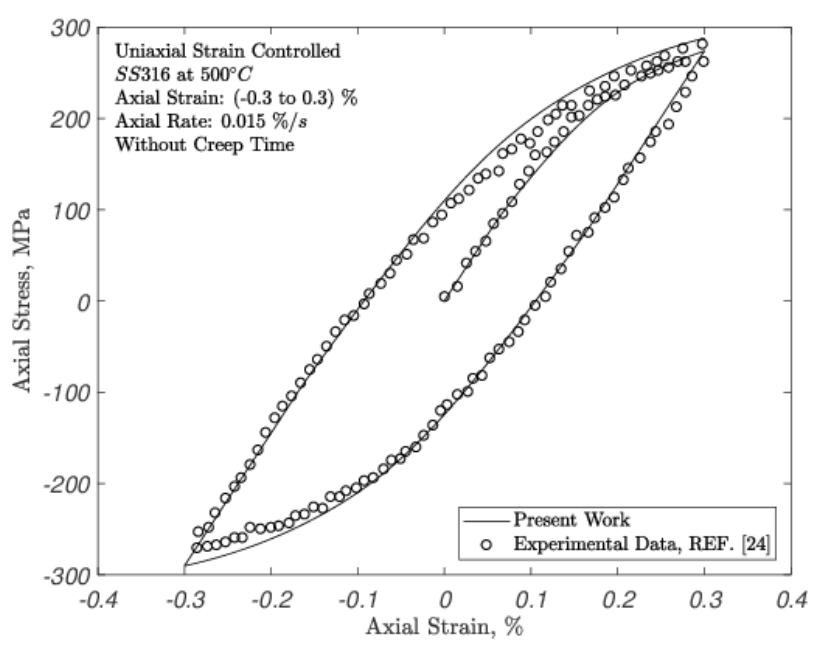

Fig. 3. Comparison of present work with the experimental data of beam [24]

The beam is under strain-controlled condition where the axial strain is cycled between $-0.3 \%$ and $0.3 \%$, and the strain rate which is used to evaluate the results is $\dot{\epsilon}_{x}=0.015 \% / \mathrm{s}$. The stress-strain curve for the first cycle of loading is shown in Fig. 3. As the figure shows, the numerical results are in close agreement with the experimental data and the percentage of error at the end of loading and unloading is approximately $3 \%$.

In the next example, a thick spherical vessel made of CS1020 steel under inside pressure cyclic loading is cycled through $P_{i}=0$ to $300 \mathrm{MPa}$. In order to verify the numerical method with the cyclic plasticity responses of thick sphere based on the Armstrong-Frederick hardening model [5], the rate of inelastic strain is considered as zero, $\dot{\epsilon}^{v p}=0$. The material properties of the sphere are: elasticity modulus $E=173.2 \mathrm{GPa}$, yield stress $\sigma_{0}=241 \mathrm{MPa}$, kinematic hardening parameters $C_{1}=280, C_{2}=0$, $a_{1}=197.5 \mathrm{MPa}, a_{2}=0$, isotropic hardening parameters $Q=0, b=0$. Fig. 4 shows that the numerical results of Ref. [7] and the numerical data of current work are in close agreement and the maximum error between these two results is less than $1 \%$.
In order to verify the proposed numerical algorithm with finite element method, a thick spherical vessel is modelled with ABAQUS software. The combined hardening model is considered in property module. This model is proposed by Chaboche and Lemaitre and the back stress due to this model is defined as

$$
\begin{aligned}
& d A_{i j}^{(k)}=C_{k}\left(\sigma_{i j}-A_{i j}\right) d \epsilon^{p}-\gamma_{k} A_{i j}^{(k)} d \epsilon^{p} \\
& A_{i j}=\sum_{k=1}^{N} A^{(k)}
\end{aligned}
$$

In Figs. 5 and 6 a thick sphere with radius ratio $\beta=1.5$ under inside pressure is cycled between 0 and $300 \mathrm{MPa}$. The material properties of this vessel are considered as the same as previous instance. By substituting the above relations in the proposed numerical algorithm and considering $\dot{\epsilon}^{v p}=0$, the plastic responses of present work are compared with the ABAQUS results in Figs. 5 and 6. The effective stress versus effective strain is illustrated in Fig. 5. Fig. 6 shows the effective plastic strain in the different radius of spherical vessel. The percentage of error between proposed numerical algorithm and FEM results for effective plastic strain, radial, and tangential stresses at different radii are shown in Tables 2-4. 


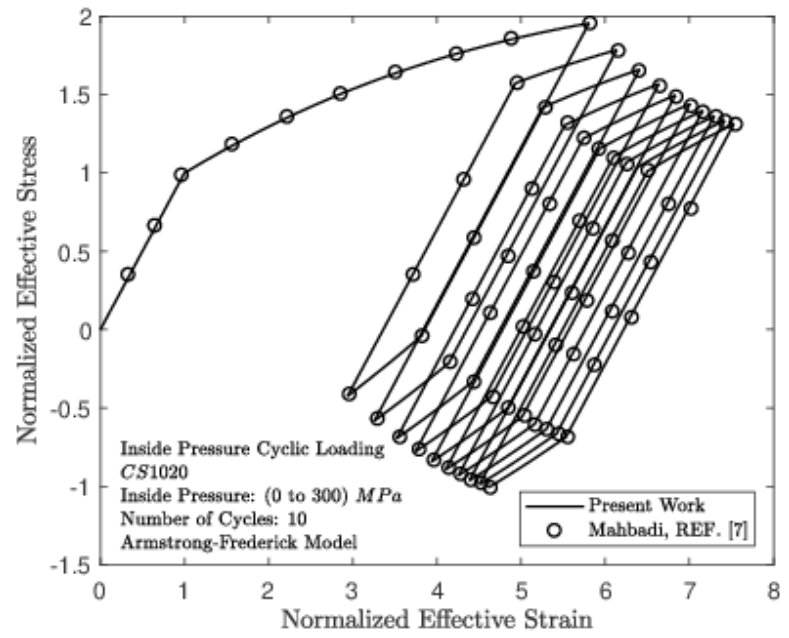

Fig. 4. Comparison of present work with the numerical results of Mahbadi et al. [7]

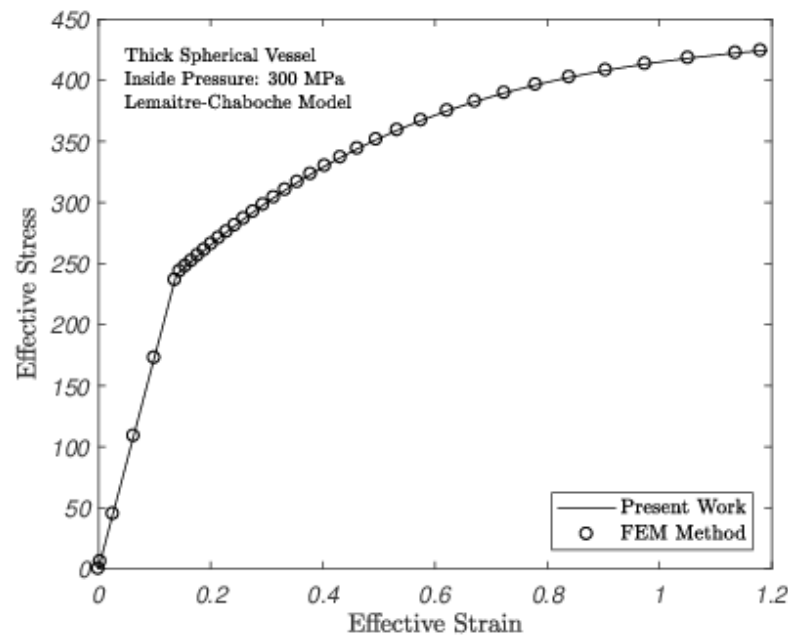

Fig. 5. Comparison of stress-strain curve with the FEM

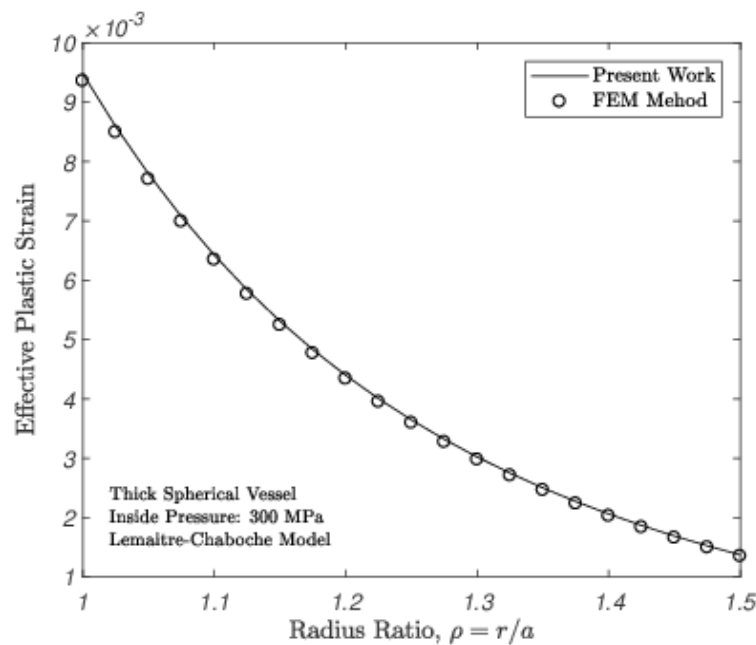

Fig. 6. Comparison of effective plastic strain versus radius ratio with the FEM 
Table 2. Comparison of effective plastic strain, $\epsilon^{p}$, with FEM results

\begin{tabular}{llll}
\hline Radius Ratio, $\beta$ & Present Work & FEM & Error, \% \\
\hline 1 & 0.009479 & 0.009355 & 1.33 \\
1.125 & 0.005836 & 0.005764 & 1.24 \\
1.25 & 0.003642 & 0.003594 & 1.37 \\
1.375 & 0.002269 & 0.002234 & 1.57 \\
1.5 & 0.001370 & 0.001347 & 1.69 \\
\hline
\end{tabular}

Table 3. Comparison of radial stress, $\sigma_{r r}$, with FEM results

\begin{tabular}{lcll}
\hline Radius Ratio, $\beta$ & Present Work & FEM & Error, \% \\
\hline 1 & -300.00 & -299.48 & 0.17 \\
1.125 & -202.93 & -202.64 & 0.15 \\
1.25 & -122.14 & -121.86 & 0.23 \\
1.375 & -55.40 & -55.22 & 0.34 \\
1.5 & 0 & 0 & 0 \\
\hline
\end{tabular}

Table 4. Comparison of tangential stress, $\sigma_{\theta \theta}$, with FEM results

\begin{tabular}{llll}
\hline Radius Ratio, $\beta$ & Present Work & FEM & Error, \% \\
\hline 1 & 124.41 & 124.46 & 0.05 \\
1.125 & 196.83 & 196.38 & 0.23 \\
1.25 & 244.96 & 244.28 & 0.28 \\
1.375 & 278.32 & 277.48 & 0.30 \\
1.5 & 303.79 & 302.78 & 0.33 \\
\hline
\end{tabular}

\subsection{Effect of loading and unloading rate}

In Figs. 7 and 8, the inside pressure of spherical vessel with radius ratio $\beta=1.5$ is cycled between 0 and $250 \mathrm{MPa}$ at uniform temperature distribution $500{ }^{\circ} \mathrm{C}$. The relaxation time after loading and unloading are considered zero. The rate of ratcheting strain, which is defined as Eq. (26), is shown in Fig. 7 for different rates of loading in $10^{\text {th }}$ cycle. As the figure shows, increasing the loading rate results into decreasing the rate of ratcheting.

$\delta \epsilon_{n^{\text {th }} \text { Cycle }}^{\text {Ratcheting }}=\epsilon_{n^{\text {th }} \text { Cycle }}^{\text {Peak }}-\epsilon_{(n-1)^{\text {th }} \text { Cycle }}^{\text {Peak }}$

\subsection{Effect of inside pressure}

In Fig. 9, the inside pressure of spherical vessel with radius ratio $\beta=1.5$ is cycled between 0 and $P_{T}$ at uniform temperature distribution $500{ }^{\circ} \mathrm{C}$. The loading and unloading times are 1 hour without creep time after loading and unloading. The rate of ratcheting strain is shown in Fig. 9 for various inside pressure in different cycles. As the figure shows, increasing the inside pressure, results into increase of the rate of ratcheting. 


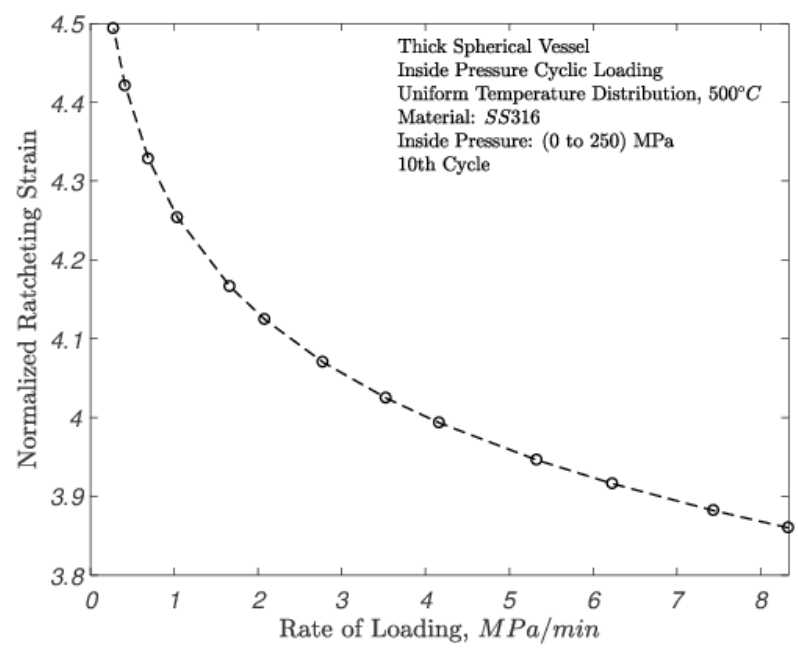

Fig. 7. Effect of loading rate on the ratcheting strain

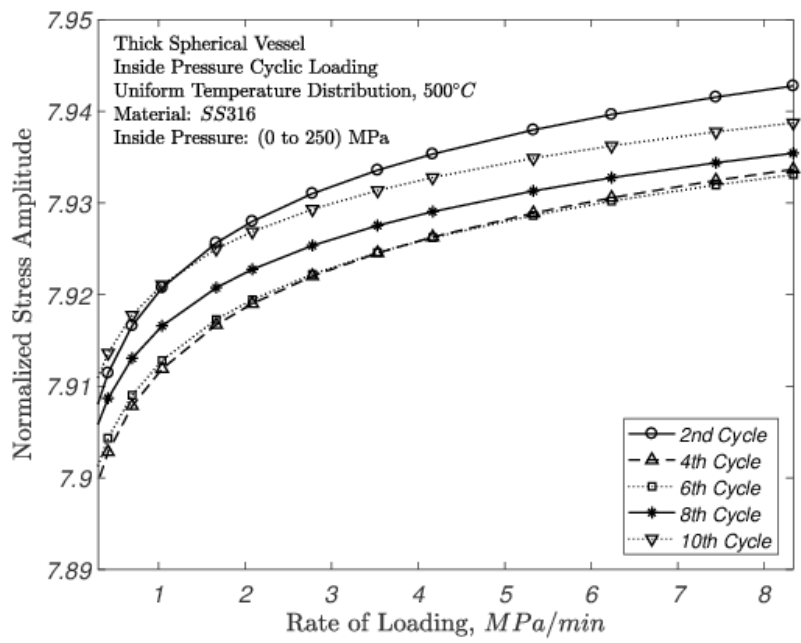

Fig. 8. Effect of loading rate on the stress amplitude

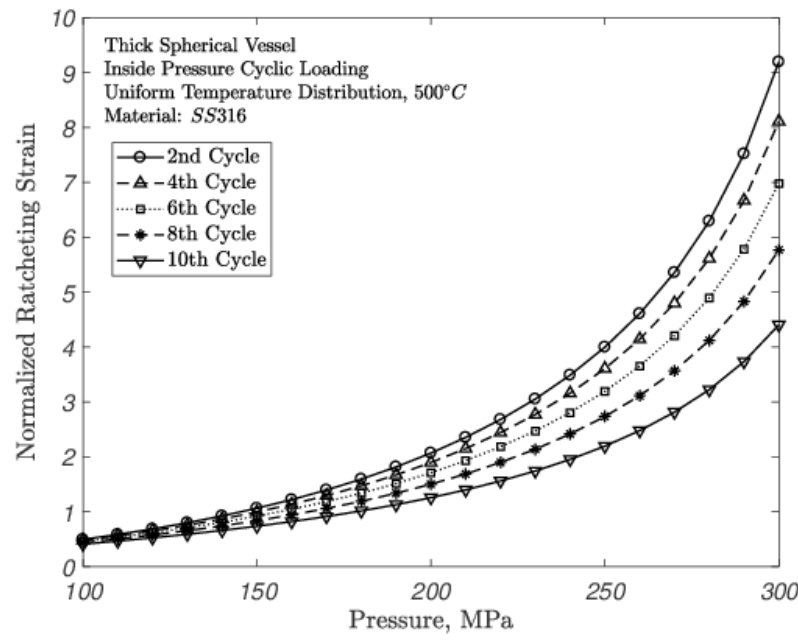

Fig. 9. Effect of insider pressure range on the ratcheting strain 


\subsection{Effect of creep time}

In Figs. 10 and 11, the inside pressure of spherical vessel with radius ratio $\beta=1.5$ is cycled between 0 and $250 \mathrm{MPa}$ at uniform temperature distribution $500{ }^{\circ} \mathrm{C}$. The rate of loading and unloading is 4.617 $\mathrm{MPa} \cdot \mathrm{min}^{-1}$. The creep time after loading is varied through 0 to $500 \mathrm{~min}$, and the responses of ratcheting rate and stress amplitude are investigated. Although increasing the creep time results to increase of ratcheting strain, the stress amplitude is decreased.

In Figs. 12 and 13, the effects of different creep time conditions for constant loading and unloading rates, considering 100 hours creep time just after the 1 st half of cycle, 100 hours creep time after each half of cycle, and without creep time is discussed. As the figures show, by adding the creep time after loading the ratcheting strain is increased while the stress amplitude is decreased. Also, adding the creep time after unloading results to increase of the ratcheting strain and slightly decrease of the stress amplitude.

\subsection{Effect of temperature}

In Figs. 14 and 15, the inside pressure of spherical vessel with radius ratio $\beta=1.5$ is cycled between 0 and $250 \mathrm{MPa}$ at uniform temperature distribution $T^{\circ} \mathrm{C}$. The temperature is varied through 300 to 600 ${ }^{\circ} \mathrm{C}$. The rate of loading and unloading is 4.167 $\mathrm{MPa} \cdot \mathrm{min}^{-1}$. The rate of ratcheting strain is shown in Fig. 14 for different temperatures in different cycles. As the figure shows, increasing the temperature distribution, results into increase of the rate of ratcheting.

Fig. 15 shows the stress amplitude versus temperature distribution in the $10^{\text {th }}$ cycle. It is shown that the stress amplitude increases by increasing the temperature.

\subsection{Effect of thickness of vessel}

In Figs. 16 and 17, the inside pressure of spherical vessel with radius ratio $\beta$ is cycled between 0 and $250 \mathrm{MPa}$ at uniform temperature distribution 500 ${ }^{\circ} \mathrm{C}$. The rate of loading and unloading is 4.167 $\mathrm{MPa} \cdot \mathrm{min}^{-1}$. The rate of ratcheting strain is shown in Fig. 16 for different thicknesses of vessel in different cycles. As the figure shows, increasing the thickness, results into exponentially decrease of the rate of ratcheting.

Fig. 17 shows the stress amplitude versus radius ratio in the $10^{\text {th }}$ cycle. It is shown that the stress amplitude is reduced by increasing the thickness of vessel.

\section{Conclusion}

A Chaboche unified viscoplastic constitutive model with combined isotropic and kinematic hardening theories is considered to estimate the ratcheting behavior of thick spherical pressure vessels under mechanical cyclic loading. Based on the mentioned hardening theory, the yield surface is transferred and the radius of yield surface is increased in the Haigh-Westergaard stress space. An accurate numerical procedure based on the modified successive approximation method is proposed to obtain cyclic responses of viscoplastic materials. The vessel is subjected to inside pressure cyclic loading at elevated uniform temperature distribution. The proposed method is applied to the governing equations in order to obtain inelastic strains and stresses. The novelty of present study, in comparison with the published papers reported on cyclic loading, is application of a time dependent constitutive model to evaluate the effects of rate on the ratcheting behavior of structures. The effects of different parameters on the ratcheting strain, accumulations strain, and the stress amplitude are investigated in this paper. The summarized results of cyclic loading analysis for a thick sphere at elevated temperatures are discussed as follows:

- Under load controlled cyclic loading, the vessel ratchets. This conclusion is reached with simple kinematic hardening models, reported in the previous researches, up to the more sophisticated models, as reported in this paper.

- Increasing the loading and unloading rates decreases the ratcheting strain, while the effective stress amplitude is increased.

- The rate of strain accumulation is increased exponentially due to increasing creep time, while the magnitude of stress amplitude is diminished. 


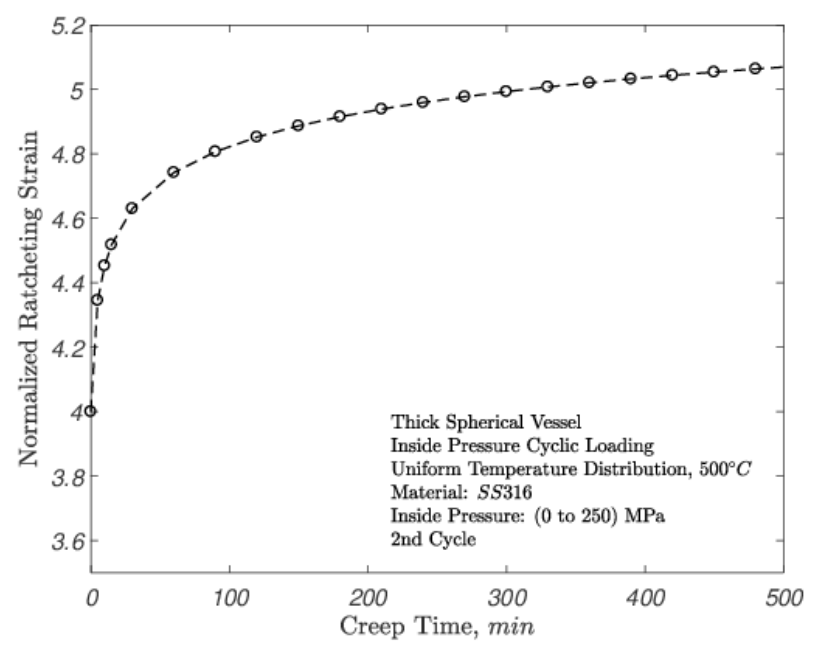

Fig. 10. Effect of creep time on the ratcheting strain

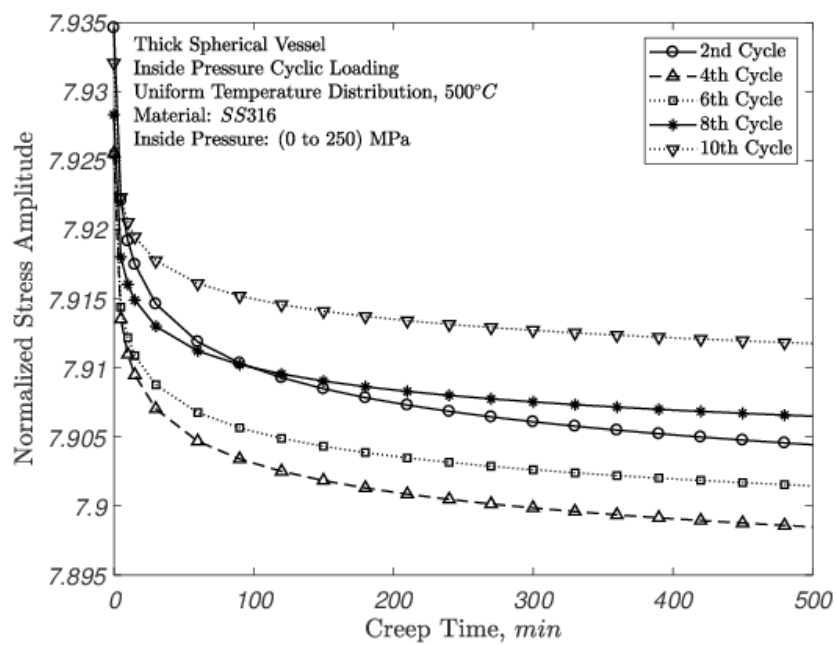

Fig. 11. Effect of creep time on the stress amplitude

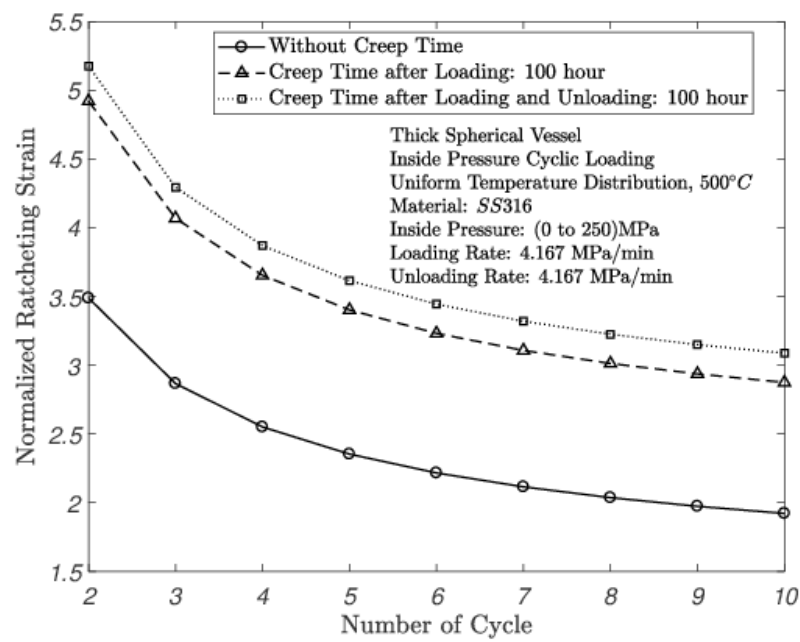

Fig. 12. Effect of different creep time conditions on the ratcheting strain 


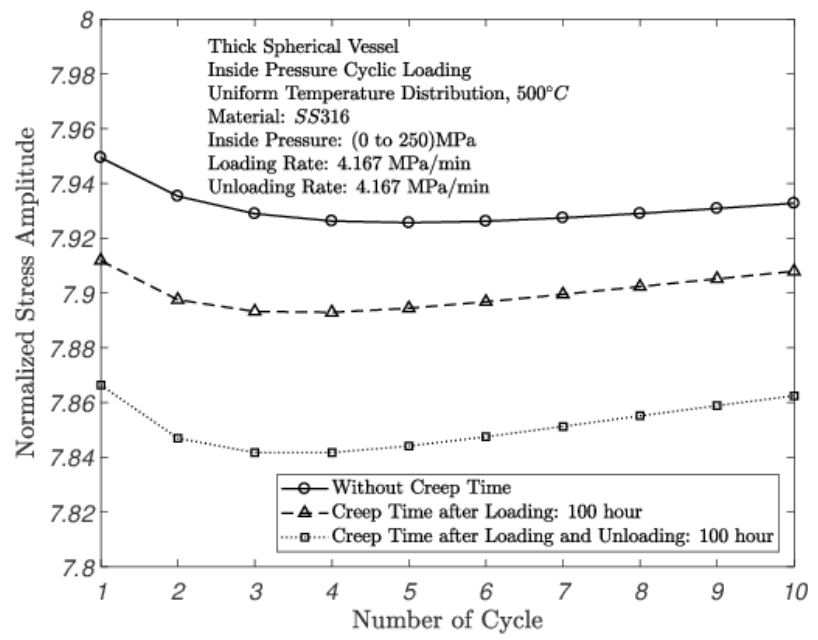

Fig. 13. Effect of different creep time conditions on the stress amplitude

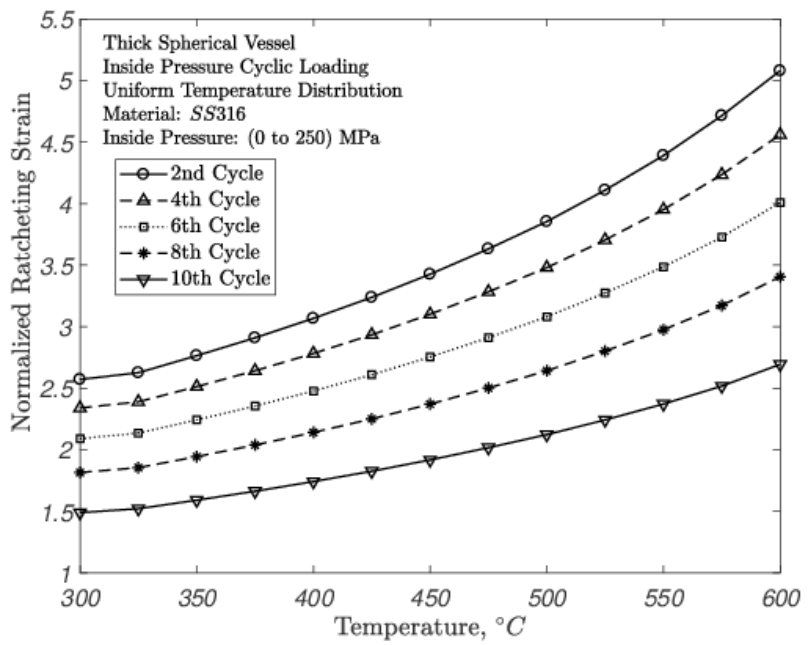

Fig. 14. Effect of temperature distribution on the ratcheting strain

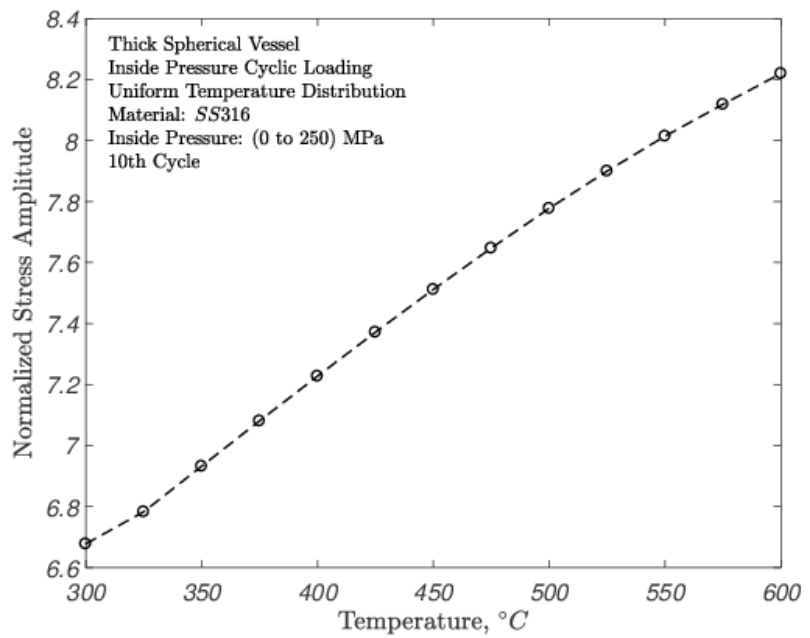

Fig. 15. Effect of temperature distribution on the stress amplitude 


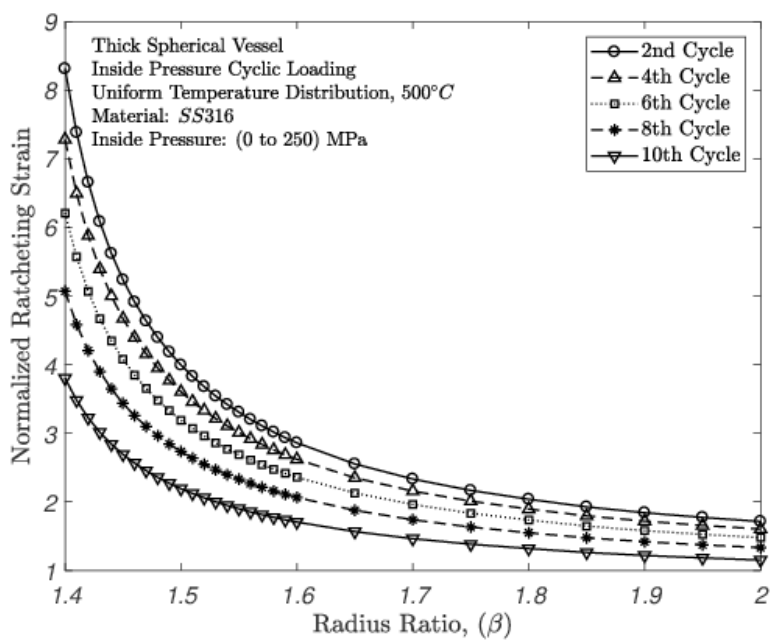

Fig. 16. Effect of thickness of vessel on the ratcheting strain

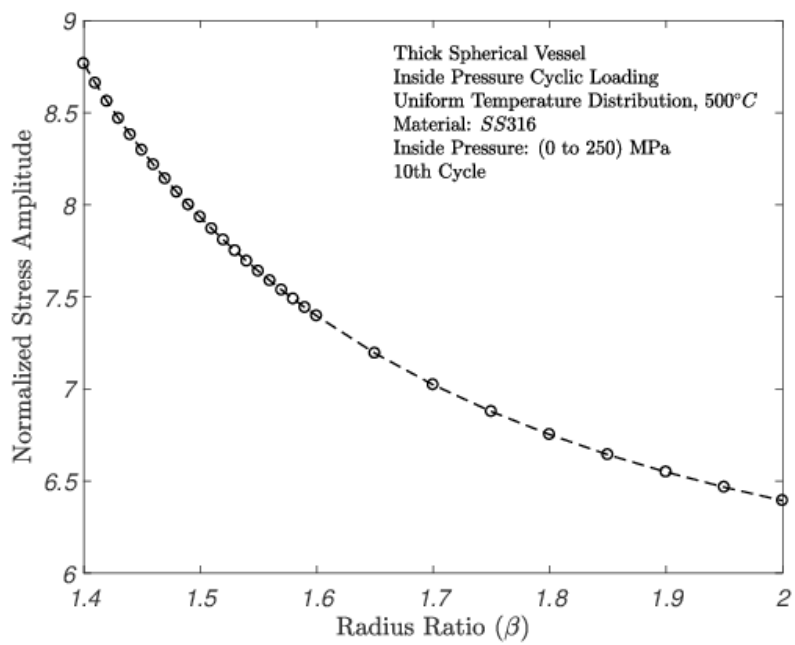

Fig. 17. Effect of thickness of vessel on the stress amplitude

- Considering relaxation time at the unloading process may raise the ratcheting strain.

- The represented data for different temperature shows that increasing this parameter increases either the ratcheting strain or the stress amplitude.

\section{Declaration of conflicting interests}

The author(s) declared no potential conflicts of interest with respect to the research, authorship, and/or publication of this article.

\section{References}

[1] Hill R. The Mathematical Theory of Plasticity. Clarendon Press, Oxfod, 1950.

[2] Westergaard H. Theory of Elasticity and Plasticity. Harvard University Press, 1952.

[3] Neale K, Shirvastava S (1990) Analytical solutions for circular bars subjected to large strain plastic torsion. ASME Journal of Applied Mechanics 57: 298-306.

[4] Prager W (1956) A new method of analyzing stresses and strains work-hardening plastic solids. ASME Journal of Applied Mechanics 23: 493-496.

[5] Armstrong P, Frederick C (1966) A mathematical representation of the multiaxial Bauschinger effect. CEGB Report No: RD/B/N 731. 
[6] Chaboche JL, Cordier G. Modelization of the strain memory effect on the cyclic hardening of 316 stainless steel. In Proceedings of $5^{\text {th }}$ International Conference on Structural Mechanics in Reactor Technology (SMiRT 5), 1979, Berlin, Germany.

[7] Mahbadi H, Eslami MR (2006) Cyclic loading of thick vessels based on the Prager and ArmstrongFrederick kinematic hardening models. International Journal of Pressure Vessels and Piping 83: 409-419.

[8] Komijani M, Eslami MR (2013) Thermal and mechanical cyclic loading of thick spherical vessels made of transversely isotropic materials. International Journal of Pressure Vessels and Piping 107: 1-11.

[9] Ejtemajou M, Eslami MR (2017) Load controlled cyclic loading of transversely isotropic cylindrical vessels based on the anisotropic kinematic hardening models. Journal of Pressure Vessel Technology 139(3): 031402 (8 pages).

[10] Chaboche J. (1986) Time-independent constitutive theories for cyclic plasticity. International Journal of Plasticity 2:149-188.

[11] Chaboche J, Rousselier G (1983) On the plastic and viscoplastic constitutive equation - Part 1: Rules developed with internal variable concept. Journal of Pressure Vessel Technology 105(2): 153-158.

[12] Chaboche JL, Rousselier G (1983) On the plastic and viscoplastic constitutive equation - Part 2: Application of internal variable concept to the 316 stainless steel. Journal of Pressure Vessel Technology 105(2): 159-164.

[13] Walker K (1981) Research and development program for non-linear structural modeling with advanced time-temperature dependent constitutive relationships. Technical Report 19820008207, NASA, United Technologies Research Center.

[14] Murakami S, Ohno N (1982) A constitutive equation of creep based on the concept of a creephardening surface. International Journal of Solids and Structures 18: 597-609.

[15] Moreno V, Jordan E (1986) Prediction of material thermomechanical response with a unified viscoplastic constitutive model. International Journal of Plasticity 2: 223-245.

[16] Ohno N (1990) Recent topics in constitutive modeling of cyclic plasticity and viscoplasticity. Journal of Applied Mechanics Reviews 43: 283295.

[17] Lee K, Krempl E (1991) An orthotropic theory of viscoplasticity based on overstress for thermomechanical deformations. International Journal of Solids and Structures 27: 1445-1459.

[18] Mahnken R, Stein E (1996) Parameter identification for viscoplastic model based on analytical derivatives of a least-squares functional and stability investigations. International Journal of Plasticity 12: 451-479.

[19] Schwertel J, Schinke B (1996) Automated evaluation of material parameters of viscoplastic constitutive equations. ASME Journal of Engineering Materials Technology 118(3): 273280.

[20] Fossum AF (1997) Parameter estimation for an internal variable model using nonlinear optimization and analytical numerical response sensitivities. ASME Journal of Engineering Materials Technology 119: 337-345.

[21] Fossum AF (1998) Rate data and material parameter estimation. ASME, Journal Engineering Materials and Technology 120: 7-12.

[22] Tong J, Vermeulen B (2004) Modelling of cyclic plasticity a viscoplasticity of a Nickel-based alloy using Chaboche constitutive equations. International Journal of Fatigue 26: 829-837.

[23] Zhan Z. A study of creep-fatigue interaction in a new nickel-based superalloy. PhD Thesis, University of Portsmouth, 2004.

[24] Gong YP, Hyde CJ, Sun W, Hyde TH (2010) Determination of material properties in the Chaboche unified viscoplasticity model. Journal of Materials Design and Applications 224: 19-29.

[25] Shojaei A, Eslami MR, Mahbadi H (2010) Cyclic loading of beams based on the Chaboche model. International Journal of Mechanics and Materials in Design 6: 217-228.

[26] Chen W, Wang F, Kitamura T, Feng M (2017) A modified unified viscoplasticity model considering time-dependent kinematic hardening for stress relaxation with effect of loading history. International Journal of Mechanical Sciences 133: 883-892.

[27] Hang L, Guang S, Gao L, Jie HWJ, Dong L, Qiang T, Cheng Z, Bo C (2019) Unified viscoplastic constitutive model under axial-torsional thermomechanical cyclic loading. International Journal of Mechanical Sciences 150: 90-102.

[28] Chen W, Kitamura T, Feng M (2018) Creep and fatigue behavior of 3161 stainless steel at room temperature: Experiments and a revisit of a unified viscoplasticity model. International Journal of Fatigue 112: 70-77. 
[29] Benaarbia A, Rouse J, Sun W (2018) A thermodynamically-based viscoelastic-viscoplastic model for the high temperature cyclic behavior of 912. International Journal of Plasticity 107: 100121.

[30] Rae Y, Benaarbia A, Hughes J, Sun W (2019) Experimental characterization and computational modelling of cyclic viscoplastic behavior of turbine steel. International Journal of Fatigue 124: 581594.

[31] Zheng X, Wang W, Guo S, Xuan F (2019) Viscoplastic constitutive modelling of the ratchetting behavior of $35 \mathrm{crmo}$ steel under cyclic uniaxial tensile loading with a wide range of stress amplitude. European Journal of Mechanics A/Solids 76: 312-320.

[32] Dong C, Yang X, Shi D, Yu H (2014) Modeling of anisotropic tensile and cyclic viscoplastic behavior of a nickel-base directionally solidified superalloy. Materials and Design 55: 966-978.

[33] Chen W, Feng M (2015) A study of a cyclic viscoplasticity model based on hyperbolic sine form for the inelastic strain rate. International Journal of Mechanical Science 101-102: 155-160.
[34] Szmytka F, Forre A, Augustins L (2015) A time increment control for return mapping algorithm applied to cyclic viscoplastic constitutive models. Finite Elements in Analysis and Design 102-103: 19-28.

[35] Kyaw ST, Rouse JP, Lu J, Sun W (2016) Determination of material parameters for a unified viscoplasticity-damage model for a $\mathrm{p} 91$ power plant steel. International Journal of Mechanical Sciences 115-116: 168-179.

[36] Ahmed R, Barret PR, Hassan T (2016) Unified viscoplasticity modeling for isothermal low-cycle fatigue and fatigue-creep stress-strain responses of Haynes 230. International Journal of Solids and Structures 88-89: 131-145.

[37] Luk-Cyr J, Paquet D, Lanteigne J, Champliaud H, Vadean A (2017) A unified plasticity methodology for rate- and temperature- sensitive alloys exhibiting a non-linear kinematic hardening behavior. Acta Mechanica Solida Sinica 30: 27-37.

[38] Xiaoan H, Qiang Z, Xiao-Guang Y, Duo-Qi S (2019) Viscoplastic analysis method of an aeroengine turbine blade subjected to transient thermomechanical loading. International Journal of Mechanical Sciences 152: 247-256. 